\title{
Colangiografia transcolecística percutânea em cães ${ }^{1}$
}

\author{
Caique A.R. Gomes ${ }^{2 *}$, Thiago P. Anacleto ${ }^{2}$, Luan G. Prado², Angela Akamatsu², \\ Leonardo J.R. Siqueira² e Rodolfo Malagó ${ }^{2}$
}

\begin{abstract}
Gomes C.A.R., Anacleto T.P., Prado L.G., Akamatsu A., Siqueira L.J.R. \& Malagó R. 2018. [Percutaneous transcholecystic cholangiography in dogs.] Colangiografia transcolecística percutânea em cães. Pesquisa Veterinária Brasileira 38(3):522-527. Centro Universitário de Itajubá, Avenida Dr. Antônio Braga Filho 687, Porto Velho, Itajubá, MG 37501-002, Brazil. E-mail: caiqueargomes@gmail.com

Obstructive biliary pathway diseases are not rare in dogs, especially those with severe clinical signs and those that need surgical treatment. Image diagnostic exams to evaluate biliary tract, like ultrasound, conventional X-ray and CT, have been used in veterinary medicine with some restrictions such as low sensibility, and in many regions unfeasibility of achievement. Due to this, most of the pacients with biliary obstruction sings are submitted to surgical procedure before an accurate diagnostic of the disease. This paper aimed to standardize and describe the percutaneous transcolecystic cholangiography in dogs that consists in the injection of contrast inside of the gall bladder guided by ultrasound. It allows to see all the bililary tract, using X-ray. The technique was performed 10 times in six clinical healthy dogs. The puncture was performed in the 9th intercostal right space near to the sternum using a 20G catheter, the dose was $1 \mathrm{ml}$ per $4 \mathrm{~kg}$. After contrast injection, serial radiographs of the abdominal region were made, in left lateral projection and ventrodorsal, the first projections were performed immediately after contrast injection and repeated after five and ten minutes. No animal showed any side effect due to the technique, this was confirmed by observation and by hepatic function evaluation. The technique was considered efficacious to visualize all the biliary tree and to see any possible obstruction in ill dogs.

INDEX TERMS: Percutaneous, transcholecystic, cholangiography, dogs, cholecystocentesis, cholestasis, biliary obstruction, diagnostic imaging, clinics.
\end{abstract}

RESUMO.- Enfermidades que acometem o sistema biliar dos cães, principalmente as relacionadas a processos obstrutivos, não são raras, sendo em sua maioria, de etiologia grave, necessitando de tratamento cirúrgico. Os métodos de diagnóstico por imagem para investigação de obstruções biliares atualmente realizados na medicina veterinária como a ultrassonografia, radiografia convencional e tomografia computadorizada, apresentam algumas restrições, como sensibilidade insuficiente, e em muitas regiões inviabilidade de realização. Desta forma, vários pacientes são submetidos a procedimentos cirúrgicos antes da obtenção do diagnóstico preciso, que caracterize e identifique o local de obstrução.

\footnotetext{
${ }^{1}$ Recebido em 21 de dezembro de 2016.

Aceito para publicação em 23 de dezembro de 2017.

${ }^{2}$ Centro Universitário de Itajubá (FEPI), Avenida Dr. Antônio Braga Filho 687, Porto Velho, Itajubá, MG 37501-002, Brasil. *Autor para correspondência: caiqueargomes@gmail.com
}

Neste contexto, o presente trabalho objetivou padronizar e descrever a técnica de colangiografia transcolecística percutânea em cães, consistindo na injeção percutânea de contraste no interior da vesícula biliar por meio de punção ecoguiada, permitindo a visualização e avaliação semiológica das vias biliares intra e extra-hepáticas, mediante projeções radiográficas. A técnica foi executada 10 vezes em seis cães clinicamente saudáveis, sendo a punção da vesícula biliar realizada através do nono espaço intercostal direito próximo ao esterno com auxílio de um cateter 20G, injetando a dose de $1 \mathrm{ml}$ de contraste para cada $4 \mathrm{~kg}$ de peso vivo. Após a injeção do contraste foram efetuadas radiografias seriadas da região abdominal, em projeção lateral esquerda e ventrodorsal, as primeiras projeções foram realizadas imediatamente após a injeção do contraste sendo repetidas após cinco e dez minutos. Nenhum dos animais apresentou quaisquer efeitos colaterais decorrentes da técnica, sendo verificados 
por meio de observação dos mesmos e provas de função hepática comparativas pré e pós procedimento. A técnica demonstrou eficácia na demonstração radiográfica da árvore biliar, possibilitando a avaliação semiológica das vias biliares intra e extra-hepáticas. Tal avaliação permite identificar, caracterizar e localizar possíveis processos obstrutivos que acometem as vias biliares na espécie canina.

TERMOS DE INDEXAÇÃO: Colangiografia, transcolecística, percutâneo, cães, colecistocentese, colestase, obstrução biliar, diagnóstico por imagem, clínica.

\section{INTRODUÇÃO}

O conjunto de pequenos ductos orgânicos com função de drenagem e transporte, que interligam o fígado e a vesícula biliar ao duodeno, compõem as vias biliares. Estes ductos conduzem a bile sintetizada no fígado para ser armazenada na vesícula biliar e posteriormente ser liberada no duodeno (Mitidieri 2009).

Segundo Dyce et al. (2010), as vias biliares se iniciam com canalículos biliares microscópicos intra-hepáticos que vão se unindo até formarem dois grandes ductos, o ducto hepático direito e esquerdo na região do hilo, os dois se confluem em um tronco comum, seguindo até o duodeno. Do tronco comum é emitido um ramo lateral tortuoso que corre até a vesícula biliar. As variações anatômicas na formação das vias biliares são frequentes, porém, raramente alteram sua função.

Em cães a vesícula biliar possui formato piriforme, se localizando entre os lobos hepáticos medial direito e quadrado, possuindo uma porção mais dilatada denominada fundo, um segmento central compondo o corpo, sofrendo estreitamento denominado colo, que culmina na formação do ducto cístico, este por sua vez se une ao ducto hepático comum formando o ducto colédoco, o maior do trato biliar (Céspedes et al. 2008).

Assim como nos humanos, as enfermidades que acometem o sistema biliar na espécie canina não são raras, apresentando em sua maioria, etiologia grave, requerendo intervenção cirúrgica. Entre elas os colangiocarcinomas, colecistíties, colangites, colelitíases e obstruções biliares (Nelson \& Couto 2015, Muramoto et al. 2012, Salomão et al. 2012).

O sinal clínico prevalente do portador de obstrução biliar total ou parcial é a icterícia. 0 paciente ictérico com suspeita de obstrução deve ser submetido a investigações diagnósticas que objetivam identificar sua etiologia e local. As alterações laboratoriais geralmente observadas em processos obstrutivos das vias biliares incluem fosfatase alcalina, alanina aminotransferase e concentrações de bilirrubina total elevadas (Cribb et al. 1988, Fossum 2014).

A ultrassonografia abdominal pode auxiliar na detecção de alguns casos de obstrução biliar. Os achados ultrassonográficos sugestivos dependem do tempo de instalação do processo obstrutivo, tipo e tamanho do agente obstrutor além do grau de obstrução, porém, achados ultrassonográficos normais não descartam a presença de obstrução (Carvalho 2014).

As técnicas de radiografia convencional não são consideradas totalmente eficazes para a identificação dos processos obstrutivos das vias biliares, uma vez que, mesmo sendo causados por cálculos, estes raramente são radiopacos, não sendo identificados na maioria das projeções radiográficas (Kealy et al. 2012).
A tomografia computadorizada é um método versátil e permite a identificação de neoplasias, cálculos, cistos, doenças inflamatórias e anomalias, possuindo aproximadamente 75\% de sensibilidade na detecção das obstruções biliares (Franchi-Teixeira et al. 1997), porém, é inacessível a grande maioria dos clínicos veterinários.

A colangiopancreatografia endoscópica retrógrada é tecnicamente difícil e raramente realizada em cães ou gatos. A injeção direta de contraste dentro dos ductos biliares dilatados, via colocação transabdominal de uma agulha fina, foi realizada em humanos, porém muito raramente nos cães (Fossum 2014).

Os animais com obstrução da via biliar extra-hepática devem ser diferenciados daqueles com colestase intra-hepática, pois obstruções extra-hepáticas podem necessitar de cirurgia, enquanto as intra-hepáticas dificilmente exigem este tratamento. As indicações para a cirurgia exploratória de animais com suspeita de obstrução extra-hepática não estão bem definidas (Fossum 2014). Desta forma, animais são submetidos a procedimentos cirúrgicos antes da obtenção de um diagnóstico acurado, que muitas vezes descartaria este método de correção.

Considerando a gravidade das colangiopatias obstrutivas, em situações de suspeita clínica desta enfermidade, baseada em exames semiológicos convencionais e provas de função hepática anormais, a avaliação semiológica das vias biliares por meio de técnicas de diagnóstico por imagem se torna obrigatória (Teplick et al. 1985). No entanto, as técnicas atualmente executadas na rotina clínica veterinária apresentam restrições, seja por sensibilidade insuficiente, ou por inviabilidade de realização.

Neste contexto, o presente trabalho objetivou padronizar e descrever a técnica de colangiografia transcolecística percutânea em cães, possibilitando a avaliação semiológica das vias biliares intra e extra-hepáticas, e o diagnóstico de processos obstrutivos que acometem as vias biliares na espécie canina.

\section{MATERIAL E MÉTODOS}

Foram utilizados seis cães (Canis familiaris), sem raça definida, três fêmeas e três machos, com idade entre cinco e nove anos, pesando entre 14,5 e $25 \mathrm{~kg}$, clinicamente saudáveis, provenientes do canil do Hospital Escola de Medicina Veterinária do Centro Universitário de Itajubá (FEPI). Este trabalho foi executado após aprovação do Comitê de Ética para o Uso de Animais em Pesquisa da FEPI, sob protocolo no $013 / 2016$.

Os animais foram submetidos a jejum hídrico por oito horas e alimentar por 16 horas, o jejum alimentar prolongado objetivou estimular o armazenamento de bile pela vesícula biliar, gerando distensão deste órgão para facilitar o procedimento de colecistocentese.

Após o período de jejum, os animais receberam anestesia geral, que consistiu em Cetamina $10 \% 5 \mathrm{mg} / \mathrm{kg}$, Xilazina $2 \% 1 \mathrm{mg} / \mathrm{kg}$, Citrato de Fentanila $0,0025 \mathrm{mg} / \mathrm{kg}$ e Sulfato de Atropina $0,022 \mathrm{mg} / \mathrm{kg}$ por via intravenosa para indução anestésica, sendo monitorizados com auxílio de um monitor multiparamétrico Mindray MEC-1000, durante todo o procedimento. Posteriormente foram posicionados em decúbito dorsal com auxílio de uma calha cirúrgica, sendo realizada tricotomia da região hipocôndrica direita, seguida de antissepsia local, utilizando Solução Alcoólica de Clorexidina 0,5\%. 
Na região do nono espaço intercostal direito próximo ao esterno foi feito um botão anestésico com $2 \mathrm{ml}$ de Cloridrato de Lidocaína $2 \%$ associado à vasoconstritor, seguido de punção ecoguiada da vesícula biliar utilizando um cateter $20 \mathrm{G}$ acoplado a uma seringa descartável contendo a dose de $1 \mathrm{ml}$ de contraste Iohexol $300 \mathrm{mg} / \mathrm{ml}$ para cada $4 \mathrm{~kg}$ de peso vivo. Após introdução do cateter, e localização do mesmo na imagem ecográfica, visualizado como uma estrutura linear hiperecogênica em relação a bile no interior da vesícula biliar, o embolo da seringa foi levemente tracionado, a fim de succionar uma pequena quantidade de conteúdo biliar, com o objetivo de confirmar a localização intravesical do cateter e consequentemente a correta punção. Para o acompanhamento ultrassonográfico do procedimento, foi utilizado o equipamento de ultrassonografia Mindray Z5 Vet com transdutor microconvexo multifrequencial Mindray 65C15EAV.

0 contraste foi injetado no interior da vesícula biliar, e o animal imediatamente encaminhado à sala de radiologia, na qual foram efetuadas radiografias seriadas da região abdominal, em projeção lateral esquerda e ventrodorsal, utilizando o equipamento de raio $\mathrm{X}$ analógico CDK Diafix AF $500 \mathrm{~mA} / 125 \mathrm{kV}$. As primeiras projeções foram realizadas imediatamente após a injeção do contraste, sendo repetidas após cinco e dez minutos. Para facilitar a visualização das estruturas contrastadas, foi realizada a técnica da radiografia negativa, que consiste no carregamento de um chassi radiográfico com uma sequência específica de filmes, sendo inserido primeiro o filme com a imagem já revelada, obtido da técnica convencional previamente realizada, sobre ele um filme não velado, seguido de um filme totalmente radioluscente, que fica em contato com o ecran da tampa do chassi. Após o carregamento este chassi é colocado sobre a mesa do equipamento de raio $\mathrm{X}$, sendo submetido a uma incidência com a técnica consistindo em $40 \mathrm{kV}$ e $5 \mathrm{mAs}$. Ao revelar o filme, este se apresenta com cores invertidas em relação ao convencional, e deve ser avaliado com auxílio de um negatoscópio.

Após o procedimento, os animais foram mantidos em jejum alimentar de seis horas, com objetivo de evitar a contração da vesícula biliar estimulada pelo alimento, com possível extravasamento de conteúdo biliar pelo orifício gerado pelo cateter. Os animais foram também mantidos em observação por um período de 24 horas, para identificar manifestações de possíveis efeitos colaterais tardios.

Para verificar a ocorrência de possíveis lesões hepáticas decorrentes da punção da vesícula biliar, foi efetuada a coleta de sangue imediatamente antes, e 48 horas após o procedimento, sendo realizadas provas de função hepática comparativas, avaliando as concentrações séricas das enzimas fosfatase alcalina (FA) e alanina aminotransferase (ALT). Foi realizado um total de 10 repetições da técnica utilizando os seis animais, sendo que em quatro animais a técnica foi executada duas vezes, a fim de se descartar qualquer erro de técnica, influência humana no exame e ocorrência de efeitos colaterais.

\section{RESULTADOS E DISCUSSÃO}

A colecistocentese, realizada pelo nono espaço intercostal direito, se apresentou tecnicamente fácil de ser executada em todos os animais, a vesícula biliar e o cateter em seu interior foram facilmente identificados na imagem ecográfica (Fig.1), não havendo dificuldade na sucção de conteúdo biliar para confirmar a punção (Fig.2).

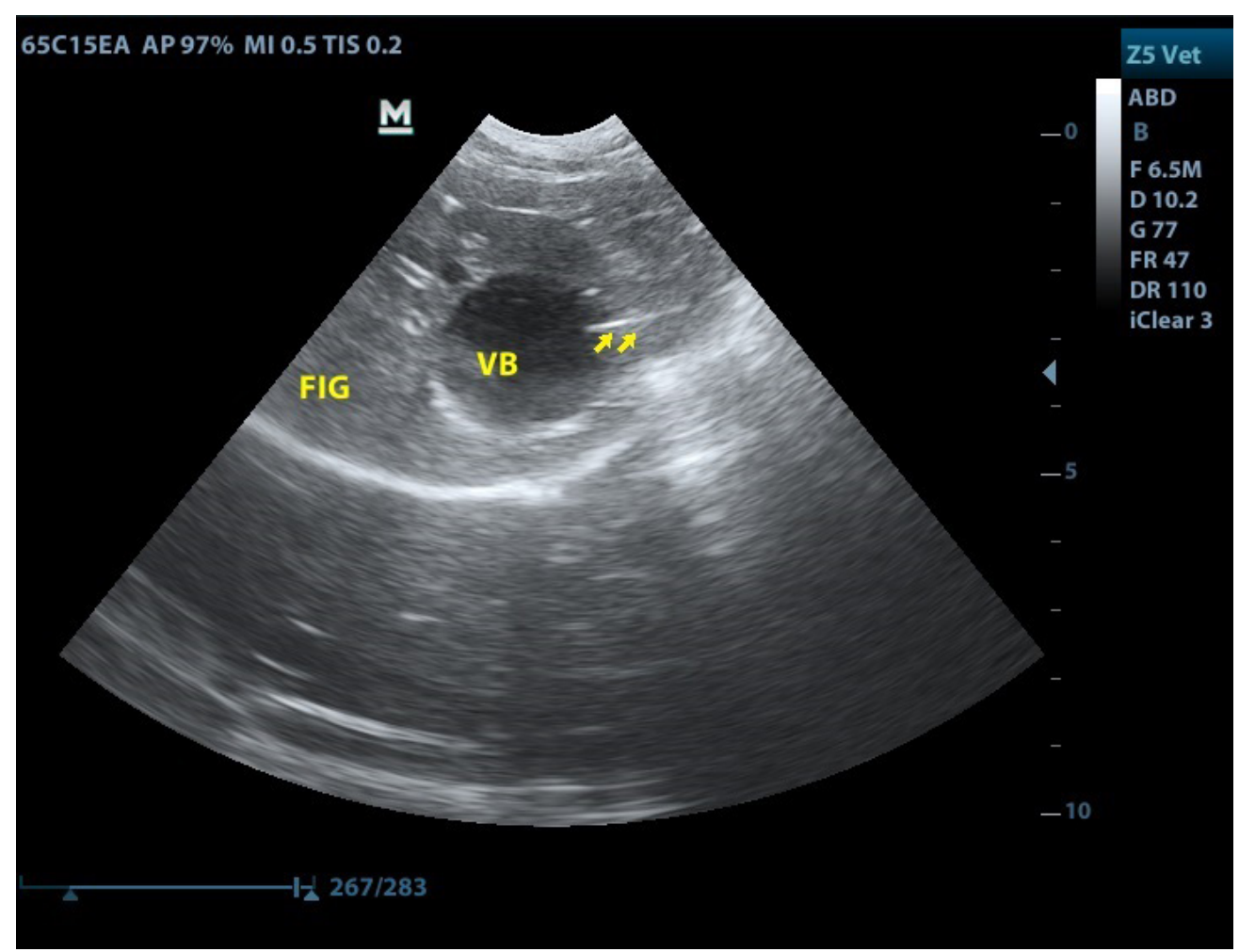

Fig.1. Imagem ecográfica do procedimento de colecistocentese, demonstrando o fígado (FIG), vesícula biliar (VB), e o cateter em seu interior (setas). 


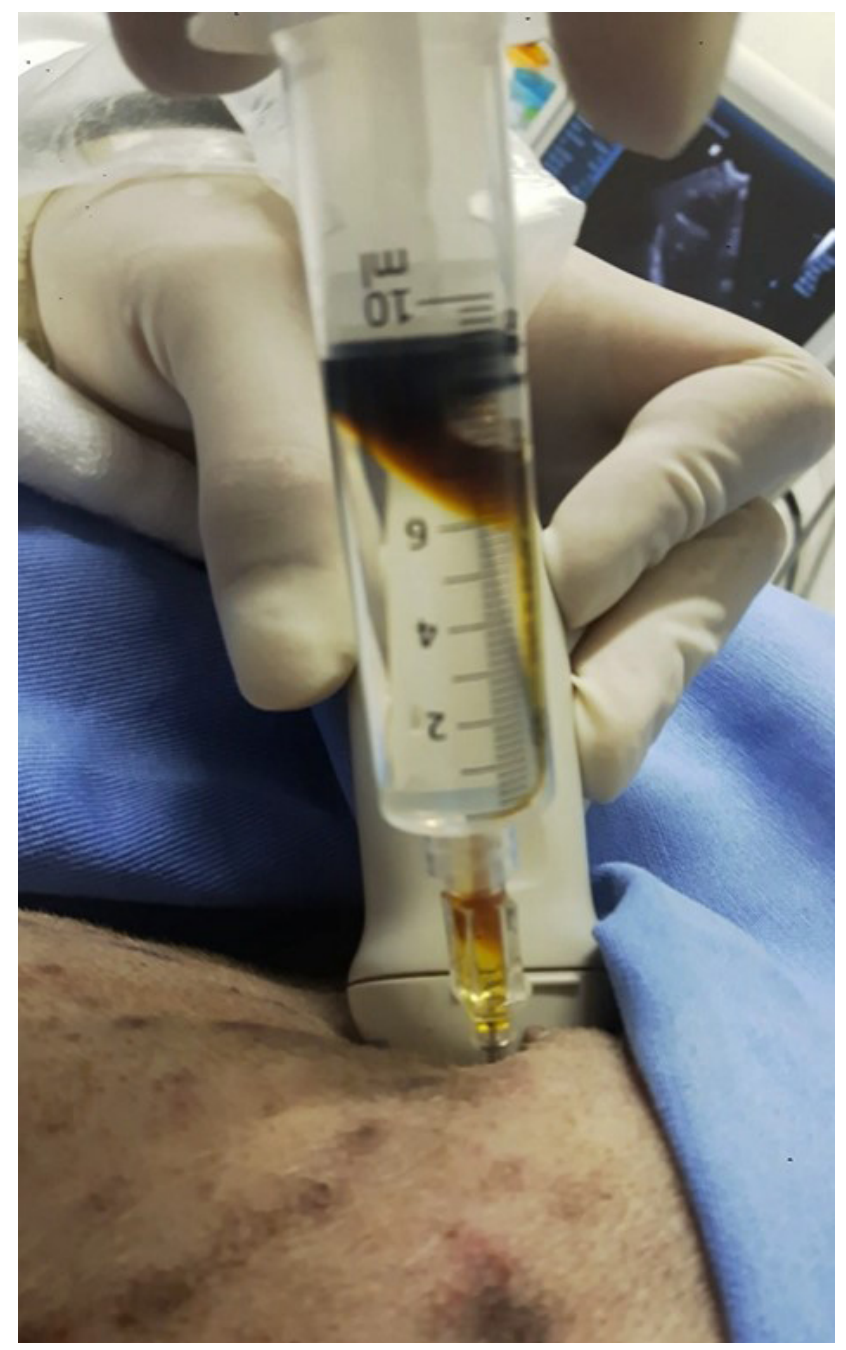

Fig.2. Procedimento de colecistocentese demonstrando a sucção de conteúdo biliar, confirmando a localização intravesical do cateter e a correta punção da vesícula biliar.

De acordo com Nelson \& Couto (2015), para se minimizar o vazamento de bile no procedimento de colecistocentese, uma agulha de pequeno calibre deve ser utilizada, sendo maiores os riscos de peritonite biliar iatrogênica quando a parede da vesícula biliar estiver gravemente acometida, o que pode ser verificado pela ultrassonografia. Neste estudo, para a realização desta técnica, foi utilizado um cateter de calibre 20G, sendo que em nenhuma projeção radiográfica foi identificado contraste extravasado na cavidade abdominal, e não foram observados efeitos colaterais decorrentes de extravasamento de bile. Antes do procedimento, a parede da vesícula biliar de cada animal foi avaliada por ultrassonografia, para a certificação de seu estado íntegro. No entanto, embora a peritonite biliar seja uma possível complicação da colecistocentese, sua ocorrência é rara, e não possui potencial suficiente para contraindicar a execução da técnica (Teplick et al. 1985).

Em procedimentos de colecistocentese realizados tanto em animais (Köster et al. 2016), quanto em seres humanos
(Saad et al. 2010), descritos na literatura, as complicações como hemorragias e extravasamento de conteúdo biliar ocorrem com maior frequência em pacientes que apresentam obstrução de ducto cístico, devido à distensão acentuada da vesícula biliar associada ao acúmulo de conteúdo em seu interior secundários ao processo obstrutivo. Por este motivo, quando houver suspeita de obstrução deste ducto, somente a dose de $1 \mathrm{ml}$ de contraste deve ser injetada.

Dabus et al. (2003), cita as reações vagais entre os efeitos colaterais da colecistostomia percutânea, devendo esta complicação ser tratada com Sulfato de Atropina e fluidos. Em nenhum dos animais utilizados neste estudo foram observadas reações vagais durante o procedimento, fato que pode ser explicado pela adição de Sulfato de Atropina no protocolo de indução anestésica, atuando na prevenção desta complicação.

Nas projeções radiográficas realizadas, foi possível identificar ductos da via biliar intra e extra-hepática, o contraste utilizado se difundiu rapidamente pela árvore biliar, sendo que nas radiografias realizadas cinco minutos após sua injeção, na maioria dos animais, se visualizava desde os canalículos biliares intra-hepáticos até a papila duodenal maior (Fig.3).

Céspedes et al. (2008), observaram que canalículos biliares provenientes do lobo quadrado do fígado se unem ao ducto cístico antes que este conflua com o ducto hepático comum, o que auxilia em sua identificação na projeção radiográfica. 0 ducto cístico possui formato tortuoso (Dyce et al. 2010), e sua forma não deve ser confundida com dilatação (Fig.4).

A identificação e avaliação dos ductos hepáticos direito e esquerdo foi facilitada nas projeções ventrodorsais, realizadas imediatamente após a injeção do contraste (Fig.5A). As projeções realizadas dez minutos após a injeção do contraste, no mesmo sentido, facilitaram a avaliação do ducto colédoco, papila duodenal maior e seu esfíncter (Fig.5B).

As concentrações séricas de FA e ALT não demonstraram alteração significativa após o procedimento, não havendo diferença estatisticamente significativa pelo teste $t(p>0.05)$, se mantendo dentro dos valores de referência para a espécie, indicando que não houve lesão hepática proveniente da punção da vesícula biliar e injeção de contraste (Quadro 1).

Embora os animais não tenham apresentado nenhum efeito colateral proveniente da técnica, não significa que são ausentes, sendo necessária a realização de mais procedimentos para a evidenciação de prováveis complicações, porém, os benefícios gerados pela técnica superam seus possíveis efeitos colaterais.

Com base na experiência obtida neste estudo, é recomendada a realização de radiografias em projeções laterais e ventrodorsais, para se minimizar falhas no diagnóstico. Além da adição de Sulfato de Atropina ao protocolo de indução anestésica, visto que pode atuar na profilaxia de possíveis reações vagais. A utilização de agulha de punção ou cateter com calibre máximo de $20 \mathrm{G}$, se mostra de grande importância, visto que diminui a probabilidade de ocorrência de extravasamento de conteúdo biliar para a cavidade abdominal e desenvolvimento de peritonite biliar iatrogênica. 


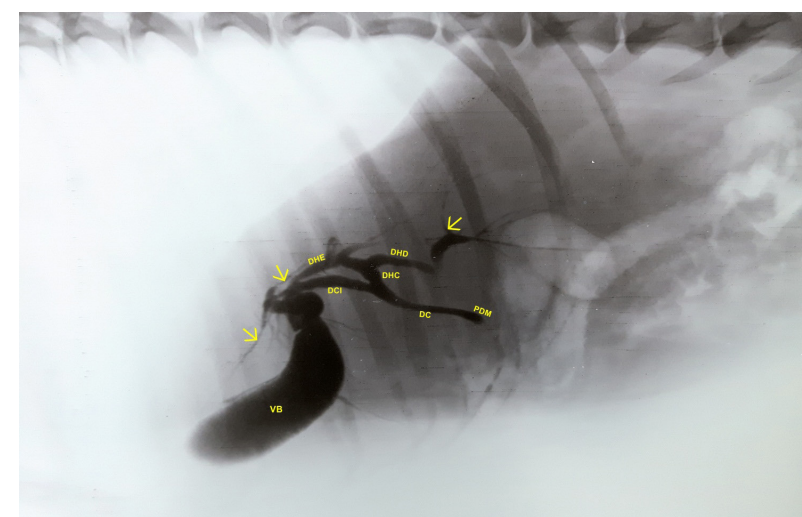

Fig.3. Fotografia de filme radiográfico submetido a técnica de radiografia negativa, em projeção lateral esquerda, demonstrando o delineamento da árvore biliar gerado pelo contraste, evidenciando a vesícula biliar (VB), canalículos biliares intra-hepáticos (setas), ducto hepático direito (DHD), ducto hepático esquerdo (DHE), ducto hepático comum (DHC), ducto cístico (DCI), ducto colédoco (DC) e papila duodenal maior (PDM).

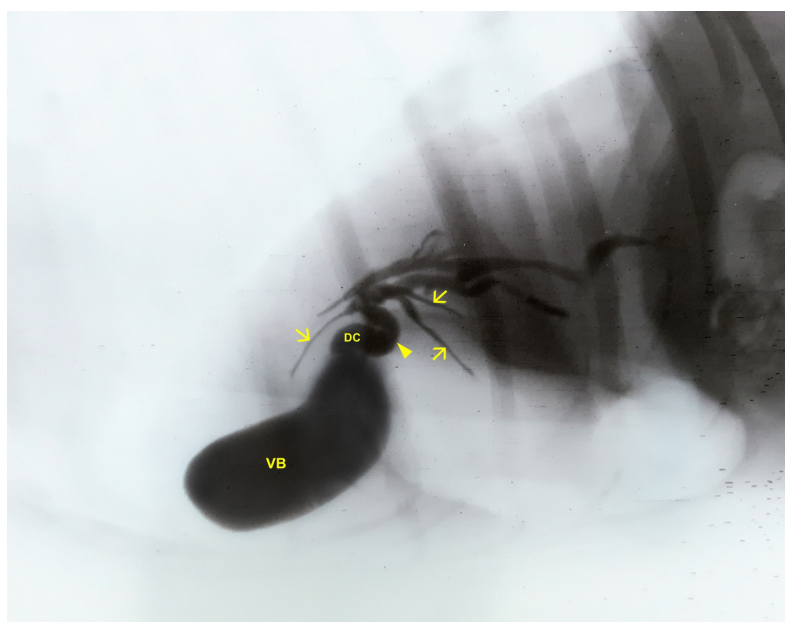

Fig.4. Fotografia de filme radiográfico submetido a técnica de radiografia negativa, em projeção lateral esquerda, evidenciando a vesícula biliar (VB), ducto cístico (DC), notar sua tortuosidade (ponta de seta), e canalículos biliares do lobo quadrado se unindo a ele (setas).

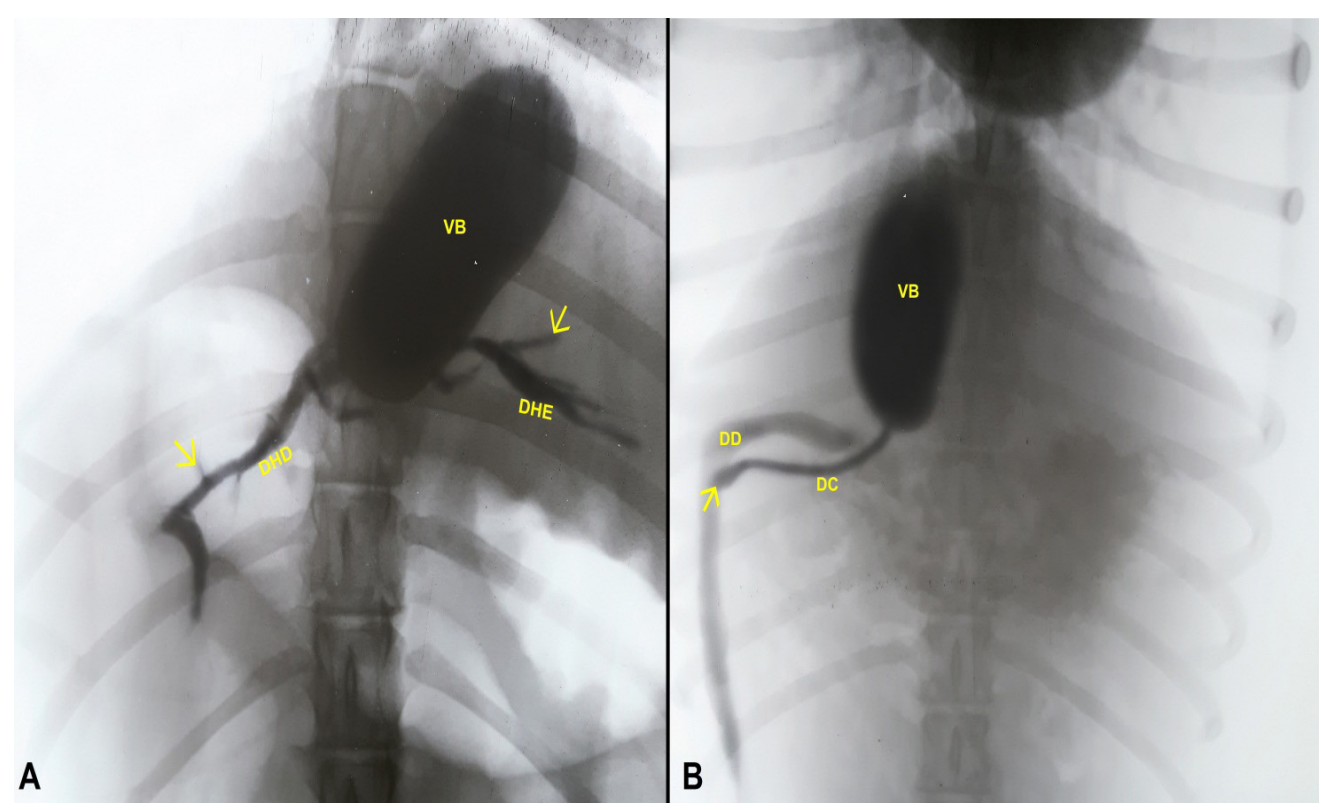

Fig.5. (A) Fotografia de filme radiográfico submetido a técnica de radiografia negativa, em projeção ventrodorsal, realizada imediatamente após a injeção do contraste, evidenciando os ductos hepáticos direito (DHD) e esquerdo (DHE), vesícula biliar (VB) e canalículos biliares intra-hepáticos (setas). (B) Projeção ventrodorsal, realizada dez minutos após a injeção do contraste, evidenciando a vesícula biliar (VB), ducto colédoco (DC), papila duodenal maior (seta), e duodeno (DD).

Quadro 1. Comparação entre as concentrações séricas de FA e ALT pré e pós realização de punção da vesícula biliar por meio da técnica de colangiografia transcolecística percutânea em cães

\begin{tabular}{|c|c|c|c|c|c|c|c|c|c|c|c|}
\hline \multirow{2}{*}{ Coleta } & \multirow{2}{*}{ Enzima } & \multicolumn{10}{|c|}{ Repetições da Técnica } \\
\hline & & $\mathrm{R} 1$ & $\mathrm{R} 2$ & R3 & $\mathrm{R} 4$ & R5 & R6 & R7 & R8 & R9 & $\mathrm{R} 10$ \\
\hline \multirow[t]{2}{*}{0 horas } & $\mathrm{FA}$ & $40,0^{\mathrm{A}}$ & $46,0^{\mathrm{A}}$ & $27,0^{\mathrm{A}}$ & $64,0^{\mathrm{A}}$ & $59,0^{A}$ & $47,0^{\mathrm{A}}$ & $33,0^{\mathrm{A}}$ & $52,0^{A}$ & $61,0^{A}$ & $49,0^{\mathrm{A}}$ \\
\hline & ALT & $42,0^{\mathrm{C}}$ & $63,0^{\mathrm{C}}$ & $33,0^{\mathrm{C}}$ & $24,0^{\mathrm{C}}$ & $70,0^{\mathrm{C}}$ & $39,0^{\mathrm{C}}$ & $68,0^{\mathrm{C}}$ & $44,0^{\mathrm{C}}$ & $29,0^{\mathrm{C}}$ & $50,0^{\mathrm{C}}$ \\
\hline \multirow[t]{2}{*}{48 horas } & FA & $37,0^{\text {в }}$ & $46,0^{\text {в }}$ & $19,0^{\text {в }}$ & $68,0^{\text {в }}$ & $61,0^{\text {в }}$ & $49,0^{\text {в }}$ & $45,0^{\text {в }}$ & $49,0^{\text {в }}$ & $56,0^{\text {в }}$ & $49,0^{\text {в }}$ \\
\hline & ALT & $47,0^{\mathrm{D}}$ & $56,0^{\mathrm{D}}$ & $40,0^{\mathrm{D}}$ & $31,0^{\mathrm{D}}$ & $68,0^{\mathrm{D}}$ & $40,0^{\mathrm{D}}$ & $63,0^{\mathrm{D}}$ & $47,0^{\mathrm{D}}$ & $31,0^{\mathrm{D}}$ & $50,0^{\mathrm{D}}$ \\
\hline
\end{tabular}

Valores de referência para a espécie canina: $\mathrm{FA}=10,0$ à 88,0U/L, ALT = 20,0 à 150,0U/L. Valores seguidos de A e B, C e D não apresentam diferença estatisticamente significativa pelo teste $t(\mathrm{p}>0.05)$. 


\section{CONCLUSÕES}

A colangiografia transcolecística percutânea é tecnicamente fácil de ser realizada nos cães, de execução rápida e relativamente indolor.

A técnica apresenta eficácia na demonstração radiográfica da árvore biliar, permitindo a avaliação semiológica das vias biliares intra e extra-hepática, tornando possível a identificação, caracterização e localização de processos obstrutivos que acometem as vias biliares na espécie canina.

Agradecimentos.- Os autores agradecem a Fundação de Amparo à Pesquisa do Estado de Minas Gerais pela Bolsa de Iniciação Científica concedida ao primeiro autor.

\section{REFERÊNCIAS}

Carvalho C.F. 2014. Fígado e vesícula biliar, p.61-89. Ultrassonografia em Pequenos Animais. $2^{\mathrm{a}}$ ed., Roca, São Paulo.

Céspedes R., Prieto E.P., Péres-Arévalo M.L., Nieves M.R., Valls V.V. \& Reyes K. 2008. Anatomía del sistema biliar del hígado en el canino. Revta Cient., Maracaibo, 18(6):667-673.

Cribb A.E., Burgener D.C. \& Reimann K.A. 1988. Bile duct obstruction secondary to chronic pancreatitis in seven dogs. Can. Vet. J. 29(8):654657. PMid: 17423102.

Dabus G.C., Dertkigil S.S.J. \& Baracat J. 2003. Percutaneous cholecystostomy: a nonsurgical therapeutic option for acute cholecystitis in high-risk and critically ill patients. Sao Paulo Med. J. 121(6):260-262. http://dx.doi. org/10.1590/S1516-31802003000600009. PMid:14989144.

Dyce K.M., Sack W.O. \& Wensing C.J.G. 2010. 0 aparelho digestório, p.100147. In: Ibid. (Eds), Tratado de Anatomia Veterinária. 4⿳亠丷a ed. Elsevier, Rio de Janeiro.
Fossum T.W. 2014. Cirurgia do sistema biliar extra-hepático, p.618-632. In:

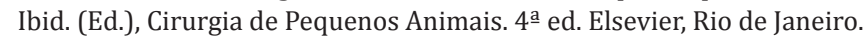

Franchi-Teixeira A.R., Antoniali F., Boin I.F.S.F. \& Leonardi L.S. 1997. Icterícia obstrutiva: diagnóstico laboratorial e de imagem. Medicina, Ribeirão Preto, 30:198-208.

Kealy J.K., McAllister H. \& Graham J.P. 2012. A vesicular biliar, p.49-50. In: Ibid. (Eds), Radiografia e Ultrassonografia do Cão e do Gato. 5a ed. Elsevier, Rio de Janeiro.

Köster L., Shell L., Illanes O., Lathroum C., Neuville K. \& Ketzis J. 2016. Percutaneous ultrasound-guided cholecystocentesis and bile analysis for the detection of Platynosomum spp. induced cholangitis in cats. J. Vet. Intern. Med. 30(3):787-793. http://dx.doi.org/10.1111/jvim.13943. PMid:27059933.

Mitidieri V.C. 2009. Anatomía de la vía biliar, p.1-11. In: Galindo F. (Ed.), Enciclopedia Cirugía Digestiva. Vol.4. Sociedad Argentina de Cirugía Digestiva. Disponível em <http://www.sacd.org.ar/enciclopedia.htm>

Muramoto C., Cesar G.C., Navarro B.C., Martin B.W., Urtado S.L.R. \& Caffalcchio L. 2012. Coleciscite enfisematosa: relato de caso. Vet. Zootec. 19(Supl.1):74-76.

Nelson R.W. \& Couto C.G. 2015. Doenças hepatobiliares e do pâncreas exócrino, p.501-628. In: Ibid. (Eds), Medicina Interna de Pequenos Animais. 5a ed. Elsevier, Rio de Janeiro.

Saad W.E.A., Wallace M.J., Wojak J.C., Kundu S. \& Cardella J.F. 2010. Quality improvement guidelines for percutaneous transhepatic cholangiography, biliary drainage, and percutaneous cholecystostomy. J. Vasc. Interv. Radiol. 21(6):789-795. http://dx.doi.org/10.1016/j.jvir.2010.01.012. PMid:20307987.

Salomão M.C., Oliveira I.N., Araujo I.M.G. \& Junior A.N. 2012. Obstrução biliar, colelitíase e colangite crônica em cão (Canis familiaris): relato de caso. Vet. Zootec. 19(Supl.1):104-106.

Teplick S.K., Haskin P.H., Pavlides C.A. \& Eykel C.V. 1985. Percutaneous transcholecystic cholangiography: experimental study. AJR Am. J. Roentgenol. 144(5):1059-1063. http://dx.doi.org/10.2214/ajr.144.5.1059. PMid:3872563. 